\title{
STATIONARY AND DYNAMIC ANALYSIS OF A WATER RELIEF VALVE
}

\author{
Paoluzzi, . $^{*}$,Borghi, . $^{\$}$, Milani, $M{ }^{\$}$ \\ * Cemoter C.N.R., Via Canal Bianco, 2844044 Cassana-Ferrara, ITALY \\ $\$$ University of Modena, Via Campi 213/b 41100 Modena, ITALY
}

\begin{abstract}
The use of water as a working fluid in hydraulic circuits is being receiving an increasing attention by both manufacturers and users, due to its environmental characteristics. Although the use of water is neither new nor innovative (more than one hundred years ago it was widely used to transfer power, and many pump and valve manufacturers have commercial product lines for water hydraulics) its introduction in the product line of a manufacturers brings many problems to the attention of the designers, from technology adaptation to material compatibility, from erosion to cavitation.

The purpose of this paper, based on a joint activity by Cemoter, University of Modena, Aron SpA and Cermet, is to show how the use of a combined approach to valve analysis can provide useful information to shorten the time to market of a valve using tap water as working fluid. Starting from an initial reminder of the basic differences between mineral oil and water in hydraulic circuits, the paper shows the results of some CFD computations on a relief valve, to evaluate the qualitative form of the pressure and velocity field, and to assess the influence of the increased turbulence on the field of motion.

In the final part, the results of a comprehensive experimental characterization of the component are presented and considered in view of the indication provided by CFD analysis, both in term of steady state characteristic curves, and dynamic response.
\end{abstract}

Keywords: Flow forces, Computational Fluid Dynamics, Hydraulic valves, Water hydraulics

\section{Introduction}

The passage from hydraulic oil to tap water as a working fluid in hydraulic circuits affects considerably the design of components both in terms of manufacturing technology (i.e. materials and surface treatment) and system performance/duration.

The most immediate effect that comes into light when approaching the design of a component intended to be used in water, is the reduced value of the viscosity that, compared to a mineral oil, is considerably lower.

As a reference, the values reported in Table 1 compare

Table 1: Comparison of oil-water properties

\begin{tabular}{|c|c|c|c|}
\hline & Water & $\begin{array}{c}\text { Mineral } \\
\text { Oil }\end{array}$ & SI Units \\
\hline \hline Density & $1 \mathrm{E} 3$ & $0.870 \mathrm{E} 3$ & $\mathrm{~kg} / \mathrm{m}^{3}$ \\
\hline $\begin{array}{c}\text { Kinematic } \\
\text { viscosity }\end{array}$ & $0.6 \mathrm{E}-3$ & 0.026 & $\mathrm{~m}^{2} / \mathrm{s}$ \\
\hline $\begin{array}{c}\text { Dynamic } \\
\text { viscosity }\end{array}$ & $6 \mathrm{E}-7$ & $0.03 \mathrm{E}-3$ & $\mathrm{~Pa}^{*} \mathrm{~s}$ \\
\hline
\end{tabular}

the density and viscosity values for a typical mineral oil and a reference tap water.
The change in physical properties has several effects, the most relevant of which is the increased amount of internal leakage in components. However, other effects exist, related to the pressure-flow rate relationship, which can affect the dynamic performance of systems.

Usually three pressure-flow relations can be used according to the fluid field of motion and geometric characteristic of the physical domain, they are the PoiseuilleHuygen, the turbulent discharge and the Blasius formulas, written respectively as:

$$
\begin{aligned}
Q & =\frac{1}{128} \frac{\pi D^{4}\left(p_{1}-p_{2}\right)}{\mu L} \\
Q & =C_{d} A \sqrt{\frac{2}{\rho}\left|\left(p_{2}-p_{1}\right)\right|} \\
Q^{1,75} & =\frac{D^{4,75}}{0,242 \mu^{0,25} \rho^{0,75}} \frac{p_{1}-p_{2}}{L}
\end{aligned}
$$

Equation 1 is valid in the case of laminar flow at small flow rates, and is characterized by the fact that a direct linear proportionality exists between pressure drop and flow rate, and an inverse proportionality with viscosity. 
This means that the leakage flow, when compared to the case of mineral oil, becomes two order of magnitude greater.

However, the situation is different when a fully turbulent efflux is considered and Equation 2 is used. In this latter case, the differences between water and mineral oil, are limited to the square root of the difference in fluid density.

This means that the situation seems to be quite clear at the extreme values of the Reynolds number range, but the situation is much more unclear at intermediate values of the Reynolds number, where other empirical formulas exist, one of which is the Blasius formula reported in Equation 3.

Generally speaking, the most relevant difference

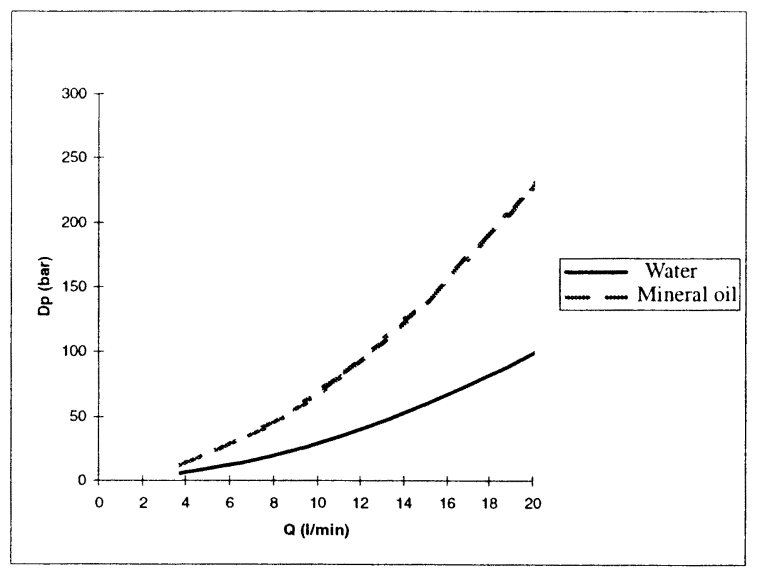

FIGURE 1 Blasius formula for water and mineral oil

between mineral oil and water is the value of the Reynolds number at given geometry and flow rate. This forces the flow field for water to become much more turbulent than that developed in oil. As a limit case it may happen that geometries well approximated by laminar pressure drop, show a considerable turbulence contribution when applied to water hydraulics. As an example, let us consider the case of a duct with circular cross section and a length to diameter ratio of 10 . Figure 1, shows the difference between oil and water using Blasius formula, which is non linear and viscosity and density dependent. The difference between the two cases are remarkable but not as high as it could be expected observing actual fluid behaviour. It must be noted that, however, the difference is in the Reynolds number of the flow, which can cause, in limit cases, the practical impossibility to apply to both fluids the same calculation formula.

\section{The role of $\mathrm{CFD}$}

The first consequence of the preliminary discussion above, is that the differences between the two cases must be analysed carefully using dedicated tools, either experimental or numerical.
The numerical tools to be used can be either non-linear simulation, to investigate the changes in dynamic behaviour, or computational fluid dynamics, to investigate the changes in flow field. When a series of numerical experiments is planned, the authors [8],[9] have shown that a special procedure aimed at non-dimensional problem statement, can allow a full characterization of a hydraulic valve, at fixed spool position, over the entire flow rate range.

Since the previous approach can also account for changes in physical properties of the fluid, the procedure can be extended and adapted to this change, in order to allow the results of a non-dimensional run, to be used for different fluids.

\subsection{Non dimensional approach reminder}

Let us remember that the general formulation of the Navier-Stokes equation is:

$$
\rho \frac{D u}{D t}=-\nabla p+\mu \nabla^{2} u
$$

where $\frac{D}{D t}$ represents the substantial derivative, $p$ is the pressure, $u$ is the velocity vector, $\rho$ and $\mu$ are respectively fluid density and kinematic viscosity.

When non-dimensional variables ${ }^{1}$ are introduced as

$$
\begin{array}{cc}
\tilde{L}=\frac{L}{L_{R}} & \tilde{u}=\frac{u}{u_{R}} \\
\tilde{t}=t \frac{u_{R}}{L_{R}} & \tilde{p}=\frac{p}{\rho u_{R}{ }^{2}}
\end{array}
$$

Equation 4 can be written in the form

$$
\frac{D u}{D t}=-\nabla p+\frac{1}{R e} \nabla^{2} u
$$

where Re is the Reynolds number of the reference flow, i.e. the Reynolds number computed using the reference values of Equation 5 and the physical properties of the reference fluid chosen (usually mineral oil). Considering that in the case under investigation, for the reasons addressed in the previous paragraph, the Reynolds number of the real flow can be with good approximation considered relatively high (or at least increasing in the case of water with respect to mineral oil), we can note that Equation 6 turns back into Equation 4 using a unit density and a viscosity equal to $1 / \operatorname{Re}[6]$.

1. the subscript $R$ refers to reference values and the nondimensional value sign $(\sim)$ will be omitted in the reminder of the text for the sake of simplicity. 
The computation of dimensional factors to be used to derive dimensional variables from the results of a nondimensional run can be limited to the case of high Reynolds number flows, and the factors are, respectively for velocity, pressure, flow rate and force:

$$
\begin{aligned}
C_{u} & =\frac{v R e}{L_{R}} & C_{p} & =\frac{\rho v^{2} R e^{2}}{L_{R}{ }^{2}} \\
C_{Q} & =v \operatorname{ReL} L_{R} & C_{F} & =\rho v^{2} R e^{2}
\end{aligned}
$$

At this point, given a run solved for a given Reynolds number, and provided that the constraints on the flow motion fully detailed in [8] are satisfied, the extension to the case of an arbitrarily different flow rate (Reynolds number), can be handled introducing the ratio

$$
\beta=\frac{R e_{1}}{R e_{0}}
$$

and deriving the new dimensional values from the old ones as

$$
\begin{aligned}
& Q_{1}=\beta Q_{0} \\
& p_{1}=\beta^{2} p_{0} \\
& F_{1}=\beta^{2} F_{0}
\end{aligned}
$$

If the $\beta$ value is varied continuously to describe the full range of flow rate of a component, a continuous characteristic curve for the component can be plotted, and the procedure can be extended to any fluid considered.

The procedure described means that non dimensional CFD runs optimized, or simply intended to be used, for a mineral oil, can be used also to investigate the behaviour of other fluids.

\section{Application to a relief valve}

The first case where this approach was applied, is a poppet relief valve, whose geometry is depicted in Figure 2. The valve acts on differential areas and the normal direction of flow is with radial inlet and axial outlet. This consideration means that the geometry considerably deviates from ideal axi-symmetric case over the inlet region, and this cause the axi-symmetric analysis approximation to be less appropriate in this case with respect to the case of axial inlet flow.

However, the main aim of the study was a qualitative analysis comparing different solutions, and therefore, provided that geometry errors are equally affecting all computations, comparative results can be considered acceptable.

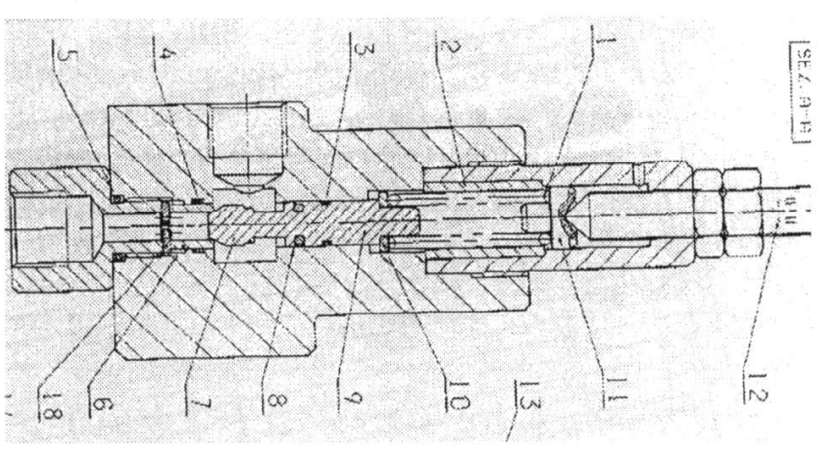

FIGURE 2 Schematic cross-section of the relief valve under study

The problem was transformed in terms of non dimensional variables using as a reference condition a flow rate of $40 \mathrm{l} / \mathrm{min}$ and a series of reference positions for the poppet, identified by its axial travel from the closure position. In the paper, the results at $1.4 \mathrm{~mm}$ are shown.

The geometry was described, represented and solved using Fidap 7.07 from Fluent, Inc.

Figure 3 shows the mesh used to solve the flow field in

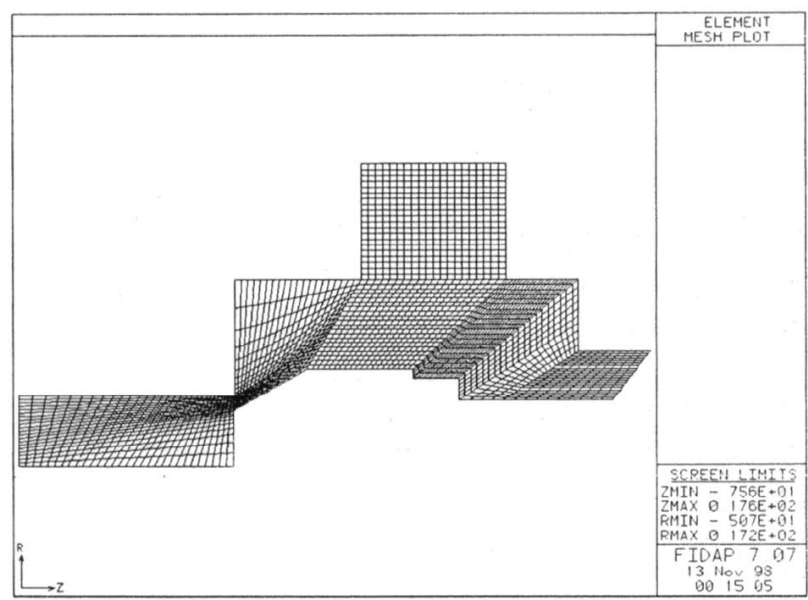

FIGURE 3 Geometry used for $1.4 \mathrm{~mm}$ opening

this case. The model is axi-symmetric and composed of approximately 40004 -node fluid elements. The turbulence effects are approximated using the well known k- $\varepsilon$ model and wall elements provided by the code were used to approximate the laminar boundary layer in turbulent flow.

As far as the boundary conditions of the flow are considered, the non dimensional values of the flow are shown in Table 2. It is worth noting that the run referred to water corresponds to a Reynolds number of 130000 , whilst the run for mineral oil is at Reynolds 2500. This difference has a limited primary effect on the flow field in terms of pressure drop and spool force due to the fact that the efflux can be considered fully turbulent. The 
Table 2: Non dimensional values used in the reference run

\begin{tabular}{c|c|c|c}
\hline Variable & Value & Unit & $\begin{array}{c}\text { Non- } \\
\text { dimension } \\
\text { al value }\end{array}$ \\
\hline Flow rate & 40 & $\mathrm{l} / \mathrm{min}$ & - \\
\hline Speed at metering & 56.5 & $\mathrm{~m} / \mathrm{s}$ & 1 \\
\hline Length & 0.0014 & $\mathrm{~m}$ & 1 \\
\hline Inlet speed & 0.77 & $\mathrm{~m} / \mathrm{s}$ & $1.37 \mathrm{E}-2$ \\
\hline
\end{tabular}

major differences should be in the $\mathrm{k}-\varepsilon$ field which must reflect the increased turbulence in the flow motion. Of course this effect could be taken into account in the redimensionalization process, when the values of the kinetic energy transfer rate and dissipation rate are converted into dimensional values. However, this process is beyond the scope of this paper and is not taken into account. The results related to the velocity field, are shown in Figure 4.

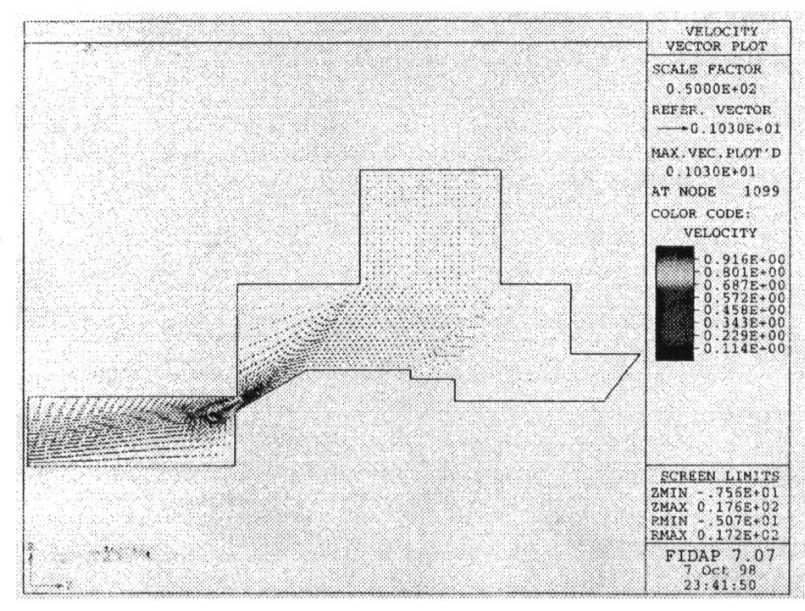

FIGURE 4 Velocity vector plot

As it is implied by the computation procedure used, the vector field does not change considerably with respect to the case of mineral oil at the same flow rate; in particular, Figure 5 shows a comparison of the continuous characteristic curves obtained for the component according to the procedure outlined in the previous paragraph.

The observation of the results in terms of $\mathrm{Q}-\Delta \mathrm{p}$ characteristic, and of $k-\varepsilon$ field distribution (not shown) allowed some preliminary considerations to be drawn up:

- overall effects, related to the mean flow of the fluid, are not affected by the transition from oil to water;

- for the geometry considered here, it is possible to estimate a discharge coefficient ranging from 0.5 to 0.52 ;

- effects related to the turbulence field, like eddies, par-

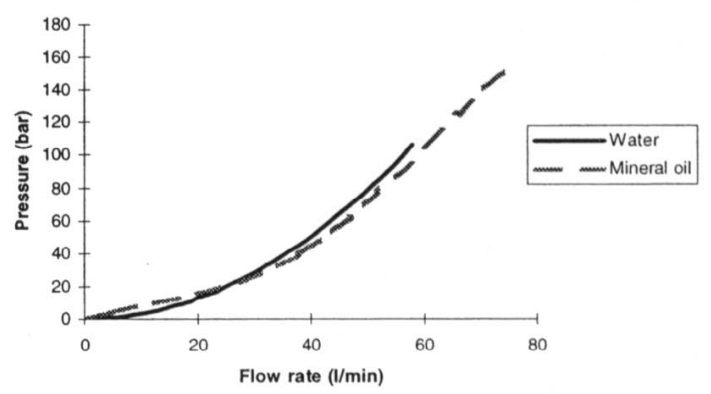

FIGURE 5 Comparison of characteristic curves for water and mineral oil as per CFD calculations

ticle transport, gas release, air trapping and cavitation vary dramatically with fluid changes.

The effect of the third point was particularly evident in the first set of experimental characterization, where noise emitted by the valve and cavitation in the fluid downstream the metering section were at unacceptable levels, although the valve performance in terms of stability, gain and precision were very good.

To compensate for this problem it was decided to act on the flow without altering the geometry of the valve, introducing a diaphragm downstream the component. It was aimed at distributing the pressure drop over two different sections, at fragmenting the large eddies into smaller ones and at accelerating the natural process of cavitation bubbles absorption.

On the numerical side this option was not investigated. It was preferred to investigate by means of CFD an option not realized in practice, in order to evaluate the opportunity of a prototype construction and test.

In this perspective, experimentation and numerical simulation become complementary, and the CFD is not used to reproduce experiments. It is used to investigate different design options, usually much more difficult, expensive and time consuming to be evaluated experimentally. This second option was an extension of the poppet in order to realize an annular channel able to distribute and equalize the flow.

The non dimensional geometry of the numerical model, together with the mesh, is shown in Figure 6.

The numerical model proved to be considerably more difficult to be solved, and the number of elements required to describe the flow field was more than three times higher than the previous case.

The solution of this case is shown in Figure 7 in terms of flow streamlines. The modification of the geometry did not prove to be effective; the major effect was the creation of new recirculation zones, without any significant effect in the reduction of the outflow turbulence and/or distribution of the pressure drop along the annular chan- 


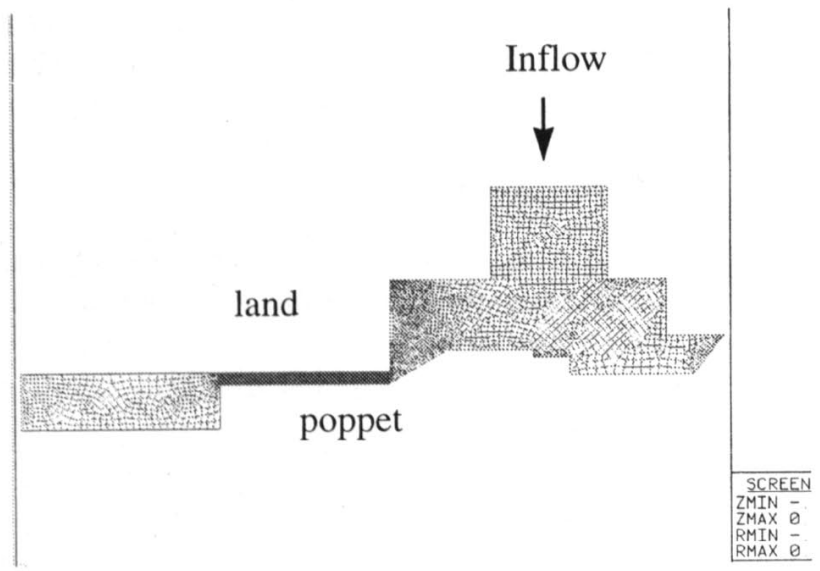

FIGURE 6 Numerical model for the modified geometry

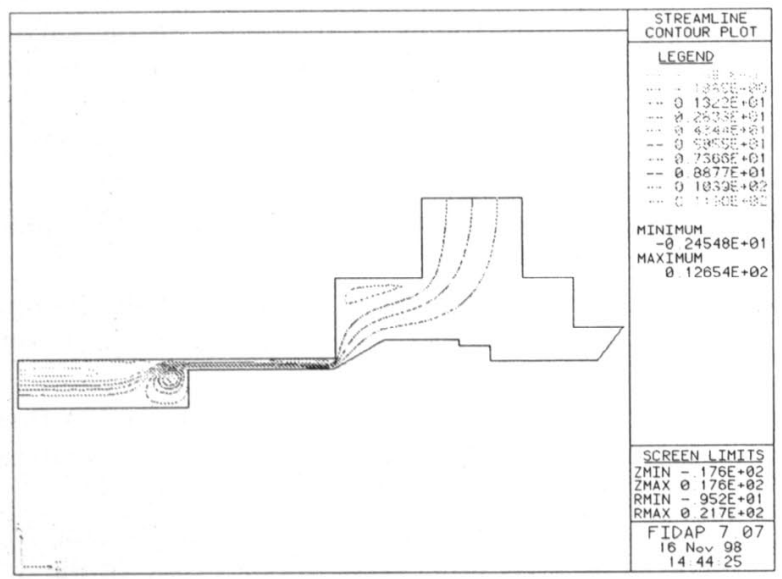

FIGURE 7 Streamlines for the modified geometry $(401 / \mathrm{min}$ $1.4 \mathrm{~mm}$ )

nel. Better performances could have been achieved with different geometries, in particular using a different ratio of inner and outer diameter, in order to force a reduction in the Reynolds number of the flow in the annular channel, but this would have required a too small gap between the two components, not practical in terms of machining precision and sensitivity to contaminants.

\section{Experimental activity}

The water relief valve just considered for CFD analysis was thoroughly investigated experimentally.

Its steady-state and dynamic response has been obtained for a wide range of operational conditions using a standard test-rig for hydraulic component characterisation (ISO 6403).

Figures 8 collects the stationary characteristic curves for a range of operating conditions spanning from 10 to 125 bar of relief pressure, and from 5 to $50 \mathrm{l} / \mathrm{min}$ of flowrate.
The valve shows hysteresis in a stationary working condition, especially at higher pressure relief valve setting values. This behaviour can be explained considering the influence of working condition on friction characteristics of seals; to limit the leakage flow between seat and spring chambers, in fact, the spool presents a du-o-ring followed by a o-ring (details n. 8 and 3, respectively, in Figure 2), whose friction response could vary significantly with the inlet pressure level and the operating lifetime.

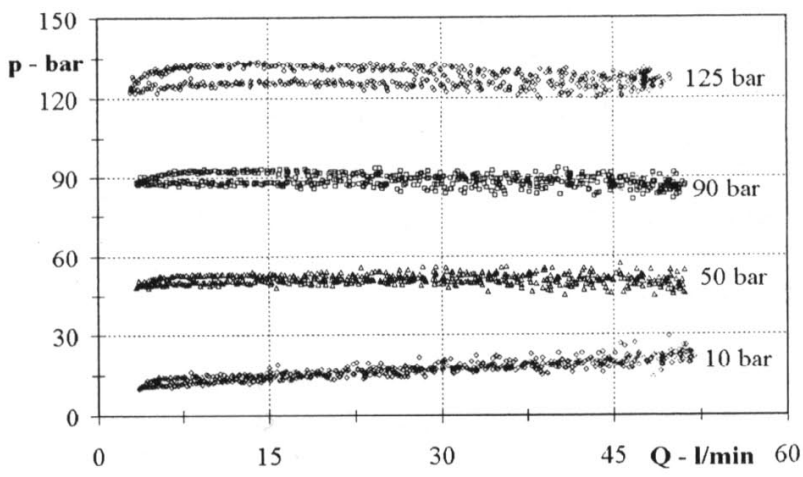

FIGURE 8 Poppet Relief Valve Characteristic Curve

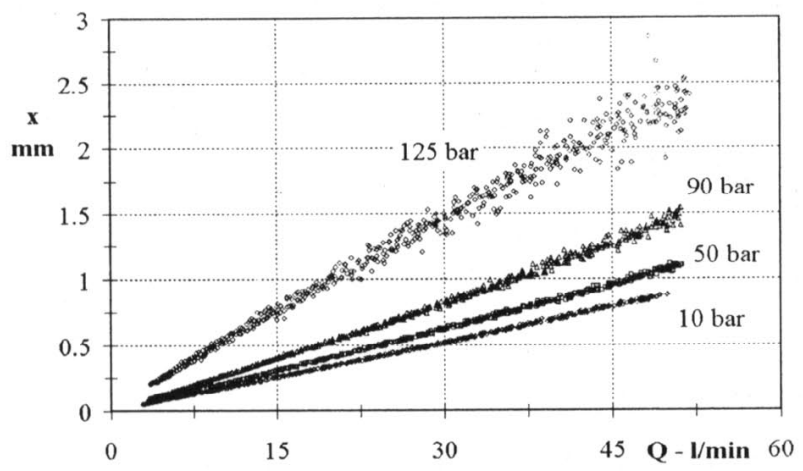

FIGURE 9 Poppet Relief Valve Operating Opening

This is made possible by the information collected in the CFD numerical experiments, and in particular the indication of a discharge coefficient approximately equal 0.5 , the knowledge of these steady-state characteristic curves lead directly to the computation of valve opening in operation.

Consequently Figure 9, reports the operating valve openings, as determined using the actual angle (30 degrees) and the actual seat diameter of the conical seat.

As shown, for each case considered the spool axial position opening can be evaluated between of 0 and $3 \mathrm{~mm}$, very far from the maximum opening allowed (about $8 \mathrm{~mm})$. 


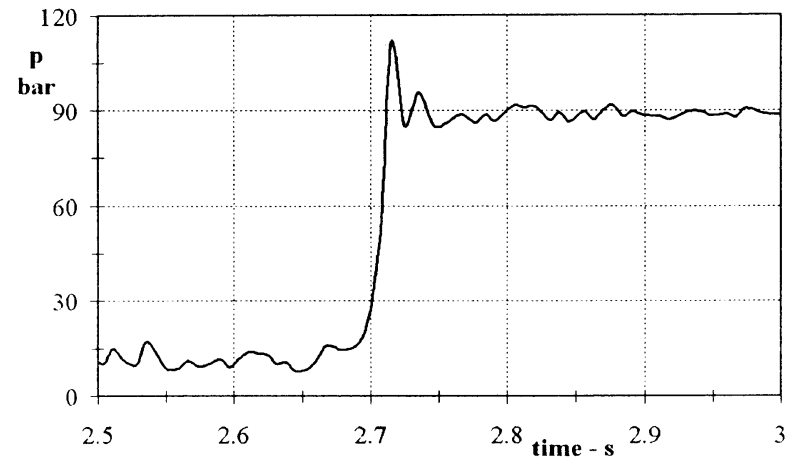

FIGURE 10 Poppet Relief Valve Dynamic Response (Relief Pressure $=90$ bar)

Finally, Figure 10 reports an example of relief valve dynamic response recorded experimentally; in this case the relief pressure was set at 90 bar.

The appearance of this curve indicates that the poppet relief valve can be considered having a satisfactory dynamic behaviour. The raising time is shorter than 0.05 $s$ and the characteristic has a limited overshoot $(15 / 20$ bar), and does not present a relevant oscillation.

\section{Conclusions}

In this paper a poppet relief valve has been considered in order to state the possibility of CFD application to water components. A simplified analysis, performed for a given internal geometry of the valve, allows some general considerations:

- overall effects, related to the mean flow of the fluid, are not affected by the transition from oil to water;

- discharge coefficient related to water flows has a lower value (0.5-0.52) with respect to mineral oil;

- effects related to the turbulence field, like eddies, particle transport, gas release, air trapping and cavitation vary dramatically changing from oil to water.

The information obtained by CFD analysis are then used to investigate a possible design modification, and to evaluate steady-state characteristic curves of the component.

As an additional example, it is shown how the use of the discharge coefficient computed by CFD simulations can be used in order to estimate the valve openings as a function of flow rate at different pressure settings.
As a final result, the design chosen for the valve was analysed experimentally in order to evaluate stationary and dynamic characteristic, proving that the overall valve behaviour satisfied the system requirements.

\section{Acknowledgements}

The authors wish to thank CERMET and Aron S.p.a. R\&D for their suggestions and technical support during experimental test.

This work was supported by Emilia Romagna Ob. II / Mis. 1.6 grant for applied research.

\section{References}

1. Blackburn, J.F., Reethof, G., Lowen Sharer, J.. Fluid Power Control, John Wiley \& Sons, 1960).

2. Lugowsky, J., Experimental investigation on the origin of flow forces in hydraulic piston valves. 10th International Conference on Fluid Power. BHR, Brugges, B, 5-7 Apr. 1993

3. Flotran 2.1 User's Manual, COMPUFLo, Charlottesville, USA.

4. FIDAP 7.07 User's Manual, Fluid Dynamics International, Evanston, IL, USA, 1993.

5. Quartapelle, L., Numerical Solution of the Incompressible Navier-Stokes Equations, Birkauser Verlag, 1993.

6. Ferretti, G., Paoluzzi, R., Zarotti, L.G., CFD flow analysis of steady-state flow forces on valves. Procs. of the 2nd ISFP Sganghai, China, 1995.

7. Palumbo, A., Paoluzzi, R., Borghi, M., Milani, M., Forces on Hydraulic Valve Spool, Procs. of the 3rd JHPS International Symposium on Fluid Power, Yokohama, Japan, 1996.

8. Borghi, M. Cantore, G., Milani, M., Paoluzzi. R., Experimental and Numerical Analysis of Forces on a Hydraulic Distributor, Procs. of the 5th Scandinavian International Conference on Fluid Power, SICFP ‘97, Vol. I, pp. 83/98, Lingkoeping, Sweden, 28/30 May, 1998.

9. Borghi, M., Cantore, G., Milani, M., Paoluzzi, R., Analysis of Hydraulic Components Using CFD Models, Procs. IMechE, Vol. 212 (7), Journal of Mechanical Engineering Science, pp. 619/629. 1998.

10. Borghi, M., Cantore, G., Milani, M., Paoluzzi, R., Transient Flow Forces Estimation on the Pilot Stage of a Hydraulic Valve, Procs. of the IMECE '98, ASME International Mechanical Engineering Congress \& Exposition, Fluid Power Systems Technology Division, Vol. 5, pp. 157/162, Anaheim, CA, USA, 15-20 November, 1998. 